\title{
Nonlinear mechanics of single-atomic-layer graphene sheets
}

\author{
Qiang Lu and Rui Huang \\ Department of Aerospace Engineering and Engineering mechanics, University of Texas, Austin, \\ TX 78712, USA
}

\begin{abstract}
The unique lattice structure and properties of graphene has drawn tremendous interests recently. By combining continuum and atomistic approaches, this paper investigates the mechanical properties of single-atomic-layer graphene sheets. A theoretical framework of nonlinear continuum mechanics is developed for graphene under both in-plane and bending deformation. Atomistic simulations are carried out to deduce the effective mechanical properties. It is found that graphene becomes highly nonlinear and anisotropic under finite-strain uniaxial stretch, and coupling between stretch and shear occurs except for stretching in the zigzag and armchair directions. The theoretical strength (fracture strain and fracture stress) of perfect graphene lattice also varies with the chiral direction of uniaxial stretch. By rolling graphene sheets into cylindrical tubes of various radii, the bending modulus of graphene is obtained. Buckling of graphene ribbons under uniaxial compression is simulated and the critical strain for the onset of buckling is compared to a linear buckling analysis.
\end{abstract}

Keywords: graphene, nonlinear elasticity, molecular mechanics 


\section{Introduction}

A monolayer of carbon (C) atoms tightly packed into a two-dimensional hexagonal lattice makes up a single-atomic-layer graphene (SALG) sheet, which is the basic building block for bulk graphite and carbon nanotubes (CNTs). Inspired by the discovery of carbon nanotubes, a series of efforts have been devoted to either grow graphene or isolate graphene from layered bulk graphite. Single and few-layered graphene have been grown epitaxially by chemical vapor deposition of hydrocarbons on metal surfaces [1-4] and by thermal decomposition of silicon carbide (SiC) [5-8]. Alternatively, thin graphene layers have been separated from intercalated graphite by chemical exfoliation [9], which however often results in sediments consisting of restacked and scrolled multilayer sheets rather than individual monolayers [10-13]. On the other hand, mechanical cleavage of graphite islands and thin films has produced graphitic plates and flakes, from just a few graphene layers to hundreds of layers [14-19]. With an improved cleavage technique, isolation of single-atomic-layer graphene was first reported in 2005 [20]. Since then, graphene has drawn tremendous interests for research in physics, materials science, and engineering [21].

Many unique properties of graphene result from its two-dimensional (2D) lattice structure. A debate however remains unsettled as to whether or not strictly 2D crystals can exist in the 3D space. Earlier theories based on the standard harmonic approximation predicted that a 2D crystal would be thermodynamically unstable and thus could not exist at any finite temperature as thermal fluctuation in the third dimension could destroy the long-range order [22, 23]. Recent experimental observations by transmission electron microscopy (TEM) and nanobeam electron diffraction revealed folding and mesoscopic rippling of suspended graphene sheets [24], suggesting that the 2D graphene lattice can be stabilized by gentle corrugation in the third 
dimension. Indeed, theoretical studies of flexible membranes [25] have led to the conclusion that anharmonic interactions between long-wavelength bending and stretching phonons could in principle suppress thermal fluctuation and stabilize atomically thin 2D membranes through coupled deformation in all three dimensions. However, the continuum membrane theory predicts severe buckling of large membranes, as the buckle amplitude scales with the membrane size. On the other hand, atomistic modeling has shown that bending of a single-atomic-layer graphene is fundamentally different from bending of a continuum plate or membrane [26, 27]. Recently, Monte Carlo simulations of equilibrium structures of SALG at finite temperatures found that ripples spontaneously form with a characteristic wavelength around $8 \mathrm{~nm}$ and the ripple amplitude is comparable to the carbon-carbon (C-C) interatomic distance ( 0.142 nm) even for very large graphene sheets [28]. The intrinsic ripples are believed to be essential for the structural stability of the 2D graphene lattice and may have major impacts on the electronic and mechanical properties of graphene.

As a new class of material, graphene offers a rich spectrum of physical properties and potential applications [21]. This paper focuses on the mechanical properties of graphene. From the micromechanical cleavage technique for isolating graphene sheets to the structural stability of the ripple morphology, the mechanical behavior of graphene has played an important role. Potential applications of graphene directly related to its mechanical properties include graphenebased composite materials [12] and nanoelectromechanical resonators [29, 30]. More broadly, development of graphene-based electronics $[4,5,17,19,21]$ may eventually require good understanding of the mechanical properties and their impacts on the performance and reliability of the devices. 
Direct measurement of mechanical properties of single-atomic-layer graphene has been challenging. An Atomic Force Microscope (AFM) was used in static deflection tests to measure the effective spring constants of multilayered graphene sheets (less than 5 layers) suspended over lithographically defined trenches, from which a Young's modulus and residual tension were extracted [31]. A similar approach was used to probe graphene sheets (no less than 8 layers) suspended over circular holes, which yielded the bending rigidity and tension by comparing the experimental data to a continuum plate model [32]. More recently, nonlinear elastic properties and intrinsic breaking strength of SALG sheets was measured by AFM indentation tests [33]. On the other hand, theoretical studies on mechanical properties of graphene have started much earlier. Even before the success of isolating and observing SALG, elastic properties of graphene have been predicted based on the C-C bond properties [26, 27, 34-37], often serving as a reference for the properties of single-walled carbon nanotubes (SWCNTs). Indeed, SWCNTs are essentially SALG rolled into cylindrical tubes, for which the mechanical properties have been studied extensively. However, the intrinsically nonlinear atomistic interactions and noncentrosymmetric hexagonal lattice of graphene dictate that mechanical properties of graphene differ from those of SWCNTs. Moreover, due to the unique 2D lattice structure, mechanical properties of graphene cannot be derived directly from its 3D form - bulk graphite. As different as they are, both CNTs and bulk graphite are made up of graphene, and thus their mechanical properties are physically related. Several recent studies have focused on mechanical properties of graphene [38-43].

In the present study, we first develop a theoretical framework for deformation of 2D graphene sheets based on nonlinear continuum mechanics in Section 2. Section 3 describes a molecular mechanics (MM) approach to simulate mechanical behavior of graphene and to extract 
its mechanical properties defined by the nonlinear continuum mechanics. Uniaxial stretch of SALG sheets is considered in Section 4, and cylindrical bending of SALG is discussed in Section 5. In Section 6, buckling of finite-width graphene ribbons is simulated and compared to a linear buckling analysis. Section 7 concludes with a summary of findings from the present study and remarks on future works.

\section{Continuum Mechanics of Two-Dimensional Sheets}

Common mechanical properties of materials such as Young's modulus, Poisson's ratio, flexural rigidity, and fracture strength are all concepts of continuum mechanics. Specifically for graphene, these properties are derived from its unique 2D lattice structure. Before establishing the connection between the atomistic structure and the mechanical properties, a theoretical framework of nonlinear continuum mechanics is developed in this section to properly define the effective mechanical properties for the 2D graphene sheets.

\subsection{Kinematics: stretch and curvature}

Take a planar graphene sheet as the reference state. The deformation of the sheet is described by a deformation gradient tensor $\mathbf{F}$ that maps an infinitesimal segment $d \mathbf{X}$ at the reference state to the corresponding segment $\mathrm{d} \mathbf{x}$ at the deformed state (Figure 1), i.e.,

$$
d \mathbf{x}=\mathbf{F} d \mathbf{X} \text { and } F_{i J}=\frac{\partial x_{i}}{\partial X_{J}}
$$


For convenience, we set up the coordinates such that $X_{3}=0$ for the graphene sheet at the reference state and thus the vector $\mathrm{d} \mathbf{X}$ has only two in-plane components $(J=1,2)$. On the other hand, $\mathrm{d} \mathbf{x}$ has three components $(i=1,2,3)$ in general.

As a measure of the deformation, the Green-Lagrange strain tensor is defined as

$$
E_{J K}=\frac{1}{2}\left(F_{i J} F_{i K}-\delta_{J K}\right)
$$

where $\delta_{J K}$ is the Kronecker delta. The deformation induces a stretch of the infinitesimal segment, namely,

$$
\lambda=\frac{|d \mathbf{x}|}{|d \mathbf{X}|}=\sqrt{1+2 E_{J K} N_{J} N_{K}}
$$

where $N_{J}=d X_{J} / d \mathbf{X} \mid$ is the unit vector in the direction of $\mathrm{d} \mathbf{X}$.

Note that, for a 2D sheet, the Green-Lagrange strain is a symmetric second-order tensor in the 2D space of the reference state, which may be decomposed into two parts:

$$
E_{J K}=E_{J K}^{P}+E_{J K}^{R}
$$

where the first term is due to in-plane displacements and the second term is due to out-of-plane rotation, as can be written in terms of the displacement components ( $\mathbf{u}=\mathbf{x}-\mathbf{X})$ :

$$
\begin{gathered}
E_{J K}^{P}=\frac{1}{2}\left(\frac{\partial u_{J}}{\partial X_{K}}+\frac{\partial u_{K}}{\partial X_{J}}+\frac{\partial u_{1}}{\partial X_{J}} \frac{\partial u_{1}}{\partial X_{K}}+\frac{\partial u_{2}}{\partial X_{J}} \frac{\partial u_{2}}{\partial X_{K}}\right) \\
E_{J K}^{R}=\frac{1}{2} \frac{\partial u_{3}}{\partial X_{J}} \frac{\partial u_{3}}{\partial X_{K}}
\end{gathered}
$$


Under an infinitesimal deformation, the nonlinear terms in the in-plane Green-Lagrange strain can be neglected, reducing to the linear strain components:

$$
E_{J K}^{P} \approx \varepsilon_{J K}=\frac{1}{2}\left(\frac{\partial u_{J}}{\partial X_{K}}+\frac{\partial u_{K}}{\partial X_{J}}\right)
$$

The nonlinear terms $E_{J K}^{R}$ may be retained for large rotation, similar to the treatment of membrane strains in the nonlinear von Karman plate theory [44].

A 2D sheet remains planar under a homogeneous deformation with a constant deformation gradient $\mathbf{F}$. An inhomogeneous deformation may induce bending and twisting of the sheet into a corrugated surface in the 3D space. The curvature of the deformed sheet can be obtained from the first and second fundamental forms of the surface in 3D [45]. Following standard procedures of differential geometry [46], we define a curvature tensor

$$
\mathrm{K}_{I J}=n_{i} \frac{\partial F_{i I}}{\partial X_{J}}=n_{i} \frac{\partial^{2} x_{i}}{\partial X_{I} \partial X_{J}}
$$

where the unit normal vector of the deformed surface is

$$
n_{i}=\frac{e_{i j k} F_{j 1} F_{k 2}}{\sqrt{\left(1+2 E_{11}\right)\left(1+2 E_{22}\right)-4 E_{12}^{2}}}
$$

For an arbitrary line segment $d \mathbf{X}$ at the reference state, the normal curvature at the deformed state is given by

$$
\kappa_{n}=\frac{\mathrm{K}_{I J} N_{I} N_{J}}{\lambda^{2}}
$$

where $N_{J}=d X_{J} /|d \mathbf{X}|$ is the unit vector in the direction of $\mathrm{d} \mathbf{X}$ and the stretch $\lambda$ is defined in Eq. (3). By solving a generalized eigenvalue problem [26, 45], two principal curvatures at each point 
can be obtained, from which the mean curvature and Gaussian curvature can be determined. We note that by Eq. (8) non-zero curvature occurs only under inhomogeneous deformation with nonzero gradients of the deformation gradient tensor (strain gradient).

For infinitesimal bending curvature, the unit normal is approximately $(0,0,1)$ and the curvature reduces to the familiar form: $\mathrm{K}_{I J}=\frac{\partial^{2} u_{3}}{\partial X_{I} \partial X_{J}}$, where $u_{3}$ is the lateral displacement normal to the plane of the sheet.

\subsection{Stress and moment tensors}

Within the theoretical framework of nonlinear hyperelasticity [47], the material property is derived from a strain energy density function that depends on the deformation gradient: $\Phi(\mathbf{F})$, under the assumption of homogeneous deformation. For a 2D sheet, we write the strain energy density as a function of the 2D Green-Lagrange strain and the curvature: $\Phi=\Phi(\mathbf{E}, \mathbf{K})$. Note that the energy density for the 2D sheet has a unit of $\mathrm{J} / \mathrm{m}^{2}$ or $\mathrm{N} / \mathrm{m}$, different from that for a 3D solid.

The 2D membrane stress (force per unit length) and moment intensity (moment per unit length) are defined as the work conjugates of the 2D Green-Lagrange strain and the curvature, respectively:

$$
S_{I J}=\frac{\partial \Phi}{\partial E_{I J}} \text { and } M_{I J}=\frac{\partial \Phi}{\partial \mathrm{K}_{I J}}
$$

The stress tensor $S_{I J}$ is analogous to the second Piola-Kirchhoff stress tensor in 3D, while the moment tensor $M_{I J}$ represents a higher order quantity associated with the curvature. 
The nominal stress acting on the 2D sheet is defined as the force at the deformed state per unit length of a line segment at the reference state, which can be obtained as the work conjugate of the deformation gradient $\mathbf{F}$, namely

$$
P_{i J}=\frac{\partial \Phi}{\partial F_{i J}}
$$

The nominal stress is analogous to the first Piola-Kirchhoff stress in 3D. Using the chain rule, the nominal stress can be related to the second Piola-Kirchhoff membrane stress and moment by

$$
P_{i J}=F_{i I} S_{I J}+\frac{\partial \mathrm{K}_{I K}}{\partial F_{i J}} M_{I K}=F_{i I} S_{I J}-n_{i} \frac{\partial M_{J K}}{\partial X_{K}}+n_{i} F_{J j}^{-1} \frac{\partial}{\partial X_{K}}\left(F_{j I} M_{I K}\right),
$$

where an inverse mapping is defined by $F_{i J} F_{J k}^{-1}=\delta_{i k}$ and thus $d \mathbf{X}=\mathbf{F}^{-1} d \mathbf{x}$. The differential relationship has a form similar to the relationship between bending moments and shear forces for continuum plates and shells [44].

\subsection{Tangent modulus}

The nonlinear elastic properties of the 2D sheet can be described in terms of tangent modulus for stretch and bending. Using the 2D second Piola-Kirchhoff membrane stress and the 2D Green-Lagrange strain, the material tangent modulus for in-plane deformation is defined as

$$
C_{I J K L}=\frac{\partial S_{I J}}{\partial E_{K L}}=\frac{\partial^{2} \Phi}{\partial E_{I J} \partial E_{K L}}
$$

Similarly, the tangent bending modulus of the sheet is

$$
D_{I J K L}=\frac{\partial M_{I J}}{\partial \mathrm{K}_{K L}}=\frac{\partial^{2} \Phi}{\partial \mathrm{K}_{I J} \partial \mathrm{K}_{K L}}
$$


In addition, there may exist a cross-term modulus for the coupling of in-plane and bending deformation:

$$
\Lambda_{I J K L}=\frac{\partial S_{I J}}{\partial \mathrm{K}_{K L}}=\frac{\partial M_{K L}}{\partial E_{I J}}=\frac{\partial^{2} \Phi}{\partial E_{I J} \partial \mathrm{K}_{K L}}
$$

Noting the symmetry in the 2D stress, stain, moment, and curvature tensors, the modulus can be written in an abbreviated matrix form by Voigt's notation, and an incremental relationship is obtained as follows:

$$
\begin{aligned}
& \left(\begin{array}{l}
d S_{11} \\
d S_{22} \\
d S_{12}
\end{array}\right)=\left[\begin{array}{lll}
C_{11} & C_{12} & C_{13} \\
C_{21} & C_{22} & C_{23} \\
C_{31} & C_{32} & C_{33}
\end{array}\right]\left(\begin{array}{c}
d E_{11} \\
d E_{22} \\
2 d E_{12}
\end{array}\right)+\left[\begin{array}{lll}
\Lambda_{11} & \Lambda_{12} & \Lambda_{13} \\
\Lambda_{21} & \Lambda_{22} & \Lambda_{23} \\
\Lambda_{31} & \Lambda_{32} & \Lambda_{33}
\end{array}\right]\left(\begin{array}{c}
d \mathrm{~K}_{11} \\
d \mathrm{~K}_{22} \\
2 d \mathrm{~K}_{12}
\end{array}\right) \\
& \left(\begin{array}{l}
d M_{11} \\
d M_{22} \\
d M_{12}
\end{array}\right)=\left[\begin{array}{lll}
D_{11} & D_{12} & D_{13} \\
D_{21} & D_{22} & D_{23} \\
D_{31} & D_{32} & D_{33}
\end{array}\right]\left(\begin{array}{c}
d \mathrm{~K}_{11} \\
d \mathrm{~K}_{22} \\
2 d \mathrm{~K}_{12}
\end{array}\right)+\left[\begin{array}{lll}
\Lambda_{11} & \Lambda_{21} & \Lambda_{31} \\
\Lambda_{12} & \Lambda_{22} & \Lambda_{32} \\
\Lambda_{13} & \Lambda_{23} & \Lambda_{33}
\end{array}\right]\left(\begin{array}{c}
d E_{11} \\
d E_{22} \\
2 d E_{12}
\end{array}\right)
\end{aligned}
$$

Equations (17) and (18) describe a generally nonlinear and anisotropic elastic behavior of a 2D sheet. Note that the coupling modulus $\Lambda_{I J K L}$ does not possess the major symmetry and thus the $\Lambda$-matrix is not symmetric, while both the C- and D-matrices are symmetric.

Under the assumption of infinitesimal deformation, Eqs. (17) and (18) reduces to linear elastic relations. For isotropic, linear elastic materials, Young's modulus and Poisson's ratio can be obtained as: $Y=C_{11}-C_{21}^{2} / C_{11}$ and $v=C_{21} / C_{11}$, respectively.

\subsection{Uniaxial stretch}

As an example, consider homogeneous deformation of a 2D sheet by a uniaxial stretch in the 1-direction, i.e, $F_{11}=\lambda$ and $F_{22}=1$, while the other components of the deformation gradient 
are all zero. The 2D Green-Lagrange strain components are then: $E_{11}=\frac{1}{2}\left(\lambda^{2}-1\right)$ and $E_{22}=E_{12}=0$, and the curvature components are all zero. Using the tangent modulus, the increments of the second Piola-Kirchhoff membrane stresses are: $d S_{11}=C_{11} d E_{11}, d S_{22}=C_{21} d E_{11}$, $d S_{12}=C_{31} d E_{11}$. By Eq. (13), the increments in the nominal stresses are:

$$
\left\{\begin{array}{l}
d P_{11}=\left(F_{11}^{2} C_{11}+S_{11}\right) d F_{11} \\
d P_{22}=\left(F_{11} C_{21}\right) d F_{11} \\
d P_{12}=\left(F_{11}^{2} C_{31}+S_{12}\right) d F_{11} \\
d P_{21}=\left(F_{11} C_{31}\right) d F_{11} \\
d P_{31}=d P_{32}=0
\end{array}\right.
$$

The nominal strain in the 1 -direction is $\varepsilon=\lambda-1$. Therefore, the tangent modulus for the nominal stress-strain is: $\bar{C}_{11}=\lambda^{2} C_{11}+S_{11}$. Only under an infinitesimal nominal strain $(\varepsilon \rightarrow 0)$, we have $\bar{C}_{11} \approx C_{11}$. The nominal shear stresses $\left(P_{12}\right.$ and $\left.P_{21}\right)$ exist when the sheet is anisotropic with nonzero $C_{31}$, which leads to the coupling between stretch and shear.

\subsection{Cylindrical bending}

Consider rolling of a 2D sheet into a cylindrical tube with a mapping $\mathbf{X} \rightarrow \mathbf{x}$ :

$$
x_{1}=R \sin \left(2 \pi \frac{X_{1}}{L}\right), x_{2}=X_{2} \text {, and } x_{3}=R-R \cos \left(2 \pi \frac{X_{1}}{L}\right)
$$

where $L$ is the width of the sheet before rolling and $R$ is the tube radius. The deformation gradient in this case is 


$$
\mathbf{F}=\left(\begin{array}{cc}
\frac{2 \pi R}{L} \cos \left(2 \pi \frac{X_{1}}{L}\right) & 0 \\
0 & 0 \\
\frac{2 \pi R}{L} \sin \left(2 \pi \frac{X_{1}}{L}\right) & 0
\end{array}\right)
$$

The corresponding 2D Green-Lagrange strain components are: $E_{11}=\frac{1}{2}\left[\left(\frac{2 \pi R}{L}\right)^{2}-1\right]$ and $E_{22}=E_{12}=0$. Therefore, the stretch in the circumferential direction of the tube is: $\lambda=\frac{2 \pi R}{L}$. In addition, by definition in Eq. (8), the curvature tensor has the components: $\mathrm{K}_{11}=\frac{4 \pi^{2} R}{L^{2}}$ and $\mathrm{K}_{22}=\mathrm{K}_{12}=0$. The normal curvature for a line segment in the circumferential direction is then, $\kappa_{n}=\frac{\mathrm{K}_{11}}{1+2 E_{11}}=\frac{1}{R}$. Thus, a variation in the tube radius simultaneously changes the stretch and the curvature: $d E_{11}=\left(\frac{2 \pi}{L}\right)^{2} R d R$ and $d \mathrm{~K}_{11}=\left(\frac{2 \pi}{L}\right)^{2} d R$.

The strain energy density of the tube can be written as: $\Phi=\Phi(R)=\Phi\left(E_{11}, \mathrm{~K}_{11}\right)$. The membrane stress in the circumferential direction and the bending moment can then be obtained as: $S_{11}=\partial \Phi / \partial E_{11}$ and $M_{11}=\partial \Phi / \partial \mathrm{K}_{11}$. By Eqs. (17) and (18), the increments of the membrane stresses and moments are: $d S_{11}=C_{11} d E_{11}+\Lambda_{11} d \mathrm{~K}_{11}$ and $d M_{11}=D_{11} d \mathrm{~K}_{11}+\Lambda_{11} d E_{11}$. Note that, while $S_{11}$ and $M_{11}$ can be obtained directly from the strain energy density function of the tube, other stress and moment components in the tube have to be evaluated separately.

\section{Atomistic Simulations of Graphene}


Atomistic simulations based on empirical potentials can be used to predict mechanical properties of materials. In the present study, we adopt a molecular mechanics (MM) approach to simulate single-atomic-layer graphene sheets subjected to uniaxial stretch, cylindrical bending, and buckling instability. The MM simulations are used to determine the static equilibrium state of graphene by minimizing the total potential energy with respect to the atomic positions. The strain energy density as well as viral stresses can be obtained directly from the MM simulations, with which the effective mechanical properties (e.g., tangent modulus) can be deduced based on the continuum mechanics theory.

The MM simulations follow the standard procedures with a few exceptions as pointed out in the subsequent sections for specific examples. For completeness, the empirical potential used in the $\mathrm{MM}$ simulations is presented in this section, along with the method for viral stress calculations.

\subsection{Empirical potential}

Several empirical potential functions describing C-C atomic interactions have been developed [48, 49], which have enabled both large-scale atomistic simulations [39, 41, 50] and closed-form predictions of the elastic properties of graphene [26, 27, 43]. In particular, the second-generation reactive empirical bond-order (REBO) potential energy for solid carbon and hydrocarbon molecules allows for covalent bond breaking and forming with appropriate changes in atomic hybridization, which has been shown to be a reliable potential function for simultaneously predicting bond energy, bond length, surface energy, and bulk elastic properties of diamond [49]. The REBO potential is used in the present study for MM simulations of graphene. 
The chemical binding energy between two carbon atoms is written in the form

$$
V_{i j}=V\left(r_{i j}\right)=V_{R}\left(r_{i j}\right)-\bar{b} V_{A}\left(r_{i j}\right)
$$

where $r_{i j}$ is the interatomic distance, $V_{R}$ and $V_{A}$ are the repulsive and attractive terms, respectively, as given by

$$
\begin{gathered}
V_{R}(r)=f_{c}(r)\left(1+\frac{Q}{r}\right) A e^{-\alpha r} \\
V_{A}(r)=f_{c}(r) \sum_{n=1}^{3} B_{n} e^{-\beta_{n} r}
\end{gathered}
$$

and $f_{c}$ is a smooth cutoff function that limits the range of the covalent interactions within the nearest neighbors, namely

$$
f_{c}(r)=\left\{\begin{array}{cc}
1, & r<D_{1}, \\
\frac{1}{2}\left(1+\cos \left[\frac{\left(r-D_{1}\right) \pi}{D_{2}-D_{1}}\right]\right), & D_{1}<r<D_{2}, \\
0, & r>D_{2} .
\end{array}\right.
$$

In addition to the pair potential terms, $\bar{b}$ is an empirical bond order function, which is a sum of three terms:

$$
\bar{b}=\frac{1}{2}\left(b_{i j}^{\sigma-\pi}+b_{j i}^{\sigma-\pi}\right)+b_{i j}^{\pi}
$$

where the first two terms depend on the local coordination and bond angles, and the third term represents the influence of radical energetics and $\pi$-bond conjugation as well as the dihedral angle for C-C double bonds. Together, the function $\bar{b}$ characterizes the local bonding environment so that the potential can to some extent describe multiple bonding states. As given in Ref. [49], the analytical forms of these functions are complicated and thus omitted here for brevity. 
The parameters for the C-C pair potential terms are: $Q=0.031346 \mathrm{~nm}, A=10953.5 \mathrm{eV}, \alpha$ $=47.465 \mathrm{~nm}^{-1}, B_{1}=12388.8 \mathrm{eV}, B_{2}=17.5674 \mathrm{eV}, B_{3}=30.7149 \mathrm{eV}, \beta_{1}=47.2045 \mathrm{~nm}^{-1}, \beta_{2}=$ $14.332 \mathrm{~nm}^{-1}, \beta_{3}=13.827 \mathrm{~nm}^{-1}, D_{1}=0.17 \mathrm{~nm}$, and $D_{2}=0.20 \mathrm{~nm}$. For a planar graphene sheet, these parameters lead to an equilibrium interatomic bond length, $r_{0}=0.142 \mathrm{~nm}$.

\subsection{Stresses by molecular mechanics}

In a discrete atomistic model, forces, rather than stresses, are used for the measure of mechanical interactions. Evaluation of stresses may be carried out based on the potential energy or directly from the forces. As defined in the continuum mechanics theory in Section 2, the 2D membrane stress $S_{I J}$ and moment $M_{I J}$ can be determined by differentiation of the strain energy density function with respect to the corresponding strain and curvature components, respectively. However, the energy method requires variation of the specific strain and curvature components independently. In the cases of uniaxial stretch, only one strain component $\left(E_{11}\right)$ is varied, and thus only one stress component $\left(S_{11}\right)$ can be determined by the energy method. To evaluate other stress components, we calculate the viral stresses based on a generalization of the virial theorem of Clausius [51]. In particular, the nominal stresses in a graphene sheet are calculated as the average membrane force over a reference area $A$ :

$$
P_{i J}=\frac{1}{2 A} \sum_{m \neq n}\left(X_{J}^{(n)}-X_{J}^{(m)}\right) F_{i}^{(m n)}
$$

where $F_{i}^{(m n)}$ is the interatomic force between atom $m$ and $n$ at the deformed state, and $X_{J}^{(m)}$ is the coordinate of the atom $m$ at the reference state. The kinetic part of the viral stresses has been neglected in the MM simulations. 


\section{Uniaxial Stretch of Graphene}

A rectangular computational cell of the graphene lattice with periodic boundary conditions is used to simulate uniaxial stretch of a single-atomic-layer graphene sheet in an arbitrary direction. The computational cell is obtained by multiple replications of the smallest rectangular unit cell with one side parallel to the direction of stretch. As illustrated in Fig. 2, to simulate uniaxial stretch of graphene in the $(2 n, n)$ direction, the unit cell contains 28 atoms in the shaded area, while the computational cell contains an integer number of the unit cells. The direction of stretch is designated by the chiral angle $\alpha$ measured counterclockwise from the zigzag direction.

Before each simulation, the graphene sheet is fully relaxed to acquire the equilibrium at the ground state with zero strain. A uniaxial stretch is then applied in two steps. First, all the atoms in the computational cell are displaced according to a homogeneous deformation with the prescribed strain, and the dimension of the computational cell is modified accordingly. Second, with the boundaries of the computational cell fixed, the atomic positions are relaxed by internal lattice relaxation to minimize the total potential energy. The internal relaxation is necessary for deformation of non-centrosymmetric lattices [43]. A standard quasi-Newton algorithm called LBFGS [52] is used for energy minimization.

Figure 3 shows the potential energy per atom as a function of the nominal strain $\varepsilon$ for a graphene sheet under uniaxial stretch in the zigzag direction $(\alpha=0)$, along with the snapshots of the atomic structures for $\varepsilon=0,0.16,0.282$ and 0.284 . The computational cell in this case contains 160 atoms, and its strain-free dimension is $2.130 \mathrm{~nm}$ (armchair direction) by $1.968 \mathrm{~nm}$ (zigzag direction). The strain is applied with an increment of 0.002. Clearly, the potential energy increases as the strain increases until it reaches a critical point where a sudden drop of the energy occurs. From the snapshots, we see that the atomic lattice is stretched uniformly (with internal 
relaxation) up to $\varepsilon=0.282$ while the lattice is fractured spontaneously at the next strain increment. The critical strain for the bond breaking in the zigzag direction is thus determined to be $\varepsilon_{f}=0.283$, which is the theoretical limit for a perfect graphene lattice under uniaxial stretch.

The strain energy density of the graphene sheet can be obtained as $\Phi=\left(V-V_{0}\right) / A_{0}$, where $V$ is the energy per atom at the deformed state, $V_{0}$ is the energy per atom at the ground state, and $A_{0}$ is the area pre atom at the ground state. The nominal stress $P_{11}$ can thus be obtained from the potential energy: $P_{11}=\frac{\partial \Phi}{\partial \varepsilon}$. Alternatively, the nominal stress as well as the other components ( $P_{22}, P_{12}$, and $\left.P_{21}\right)$ can be calculated from the viral stresses as given in Eq. (27). Figure 4a shows the nominal stress-strain curves for graphene under uniaxial stretch in the zigzag direction. The nominal stress $P_{11}$ obtained from the potential energy agrees closely with the corresponding viral stress. In the perpendicular direction, the nominal stress $P_{22}$ is positive, a result of the Poisson's effect. Both the shear components of the nominal stress are zero, indicating no coupling between shear and stretch in the zigzag direction.

With the nominal stress components known, the second Piola-Kirchhoff membrane stress components are evaluated by the relationship in Eq. (13). For uniaxial stretch, we have

$$
S_{11}=\frac{P_{11}}{1+\varepsilon}, S_{22}=P_{22}, S_{12}=\frac{P_{12}}{1+\varepsilon}, S_{21}=P_{21}
$$

Noting the symmetry, $S_{12}=S_{21}$, the nominal shear stresses must satisfy the relation, $P_{12}=P_{21}(1+\varepsilon)$, which is required by balance of the angular momentum. Figure $4 \mathrm{~b}$ plots the second Piola-Kirchhoff membrane stresses as a function of the Green-Lagrange strain $\left(E_{11}=\varepsilon+\varepsilon^{2} / 2\right)$, called S-E curves hereafter. 
The tangent moduli $C_{11}$ and $C_{21}$ are determined from the slopes of the S-E curves, as plotted in Fig. 5. Both the tangent moduli decrease as the strain increases, demonstrating the nonlinear elastic behavior of graphene under the uniaxial stretch. Recently, based on AFM indentation experiments on suspended single-atomic-layer graphene and a nonlinear elastic membrane model, Lee et al. [33] deduced a quadratic stress-strain relationship for graphene under uniaxial stress

$$
S_{11}=Y^{2 D} E_{11}+D^{2 D} E_{11}^{2}
$$

where $Y^{2 D}=340 \pm 50 \mathrm{~N} / \mathrm{m}$ is the 2D Young's modulus at infinitesimal strain and $D^{2 D}=-690 \pm$ $120 \mathrm{~N} / \mathrm{m}$ is a third-order elastic modulus. The negative value of $D^{2 D}$ leads to lessening of the tangent Young's modulus $\left(d S_{11} / d E_{11}=Y^{2 D}+2 D^{2 D} E_{11}\right)$ at increasingly tensile strain, similar to the tangent moduli shown in Fig. 5. However, due to the nonlinearity, the tangent moduli under uniaxial stretch cannot predict the tangent Young's modulus under uniaxial stress. Only under infinitesimal strain, the Young's modulus can be obtained as $Y=C_{11}-C_{21}^{2} / C_{11}$. As noted in the previous studies [26, 43], the Young's modulus of graphene predicted by the Brenner's potential (see Table I) is considerably lower than that predicted by $A b$ initio models [34]; the latter is close to the experimentally measured $Y^{2 D}$.

The nonlinear finite deformation of the graphene sheet breaks the hexagonal symmetry of the un-deformed lattice, leading to an anisotropic mechanical behavior. As shown in Fig. 6 for graphene sheets under uniaxial stretch in four different directions, both the tangent modulus and the fracture stress/strain vary with the direction of stretch. The tangent moduli for the four directions are plotted in Fig. 7 as functions of the Green-Lagrange strain. Only at infinitesimal strain $\left(E_{11} \rightarrow 0\right)$ the graphene is isotropic, with $C_{11}=288.8 \mathrm{~N} / \mathrm{m}, C_{21}=114.9 \mathrm{~N} / \mathrm{m}$, and $C_{31}=0$. 
These values agree closely with analytical results from previous studies using the same empirical potential [26, 27]. For uniaxial stretch in all directions, $C_{11}$ and $C_{21}$ decrease with increasing strain and become negative before the lattice is fractured spontaneously. This may seem to be surprising, but it is understood that the condition for spontaneous fracture of the graphene lattice is zero slope in the nominal stress-strain curves. As discussed in Section 2.4, the tangent modulus for the nominal stress-strain under uniaxial stretch is $\bar{C}_{11}=\lambda^{2} C_{11}+S_{11}$, where $\lambda=1+\varepsilon$. Setting $\bar{C}_{11}=0$ leads to a negative tangent modulus $C_{11}$ at the point of spontaneous fracture.

Figure 7c shows that the tangent modulus $C_{31}$ becomes nonzero under finite stretch in a direction other than zigzag ( $\alpha=0)$ or armchair $\left(\alpha=30^{\circ}\right)$. Consequently, a shear stress has to be applied to the graphene sheet in order to maintain uniaxial stretch in the chiral direction $\left(0<\alpha<30^{\circ}\right)$. The stretch-shear coupling of the planar graphene sheet may have contributed to the previously reported tension-torsion coupling of single-walled CNTs [53, 54]. Indeed, only CNTs with chirality other than zigzag or armchair were found to exhibit the coupling between tension and torsion.

Figure 8 plots the nominal fracture strain and fracture stress of graphene under uniaxial stretch versus the chiral angle of stretch. It is noted that, while the nominal fracture strain varies significantly from 0.178 to 0.283 , the nominal fracture stress varies slightly from $30.5 \mathrm{~N} / \mathrm{m}$ to 35.6 N/m. The MM simulations show that a perfect graphene lattice has the maximum fracture strain and fracture stress in the zigzag direction ( $\alpha=0)$. A minimum fracture strength seems to exist in a direction between $\alpha=19.11^{\circ}$ and $\alpha=30^{\circ}$ (armchair). The intrinsic strength of the suspended single-atomic-layer graphene sheets as determined by Lee et al. [33] based on AFM indentation experiments and a nonlinear membrane model was $42 \mathrm{~N} / \mathrm{m}$, with an isotropic fracture 
strain at 0.25 . While the fracture stress is noticeably higher, the fracture strain is well within the range of the present MM results. The relatively lower fracture stresses in Fig. 8 can be expected as a result of the relatively lower elastic modulus predicted by the Brenner's potential.

\section{Cylindrical Bending of Graphene Sheets}

It has been found that bending of single-atomic-layer graphene sheets is fundamentally different from the classical theory of plates or shells [26, 27]. Since the thickness of the graphene sheet is essentially zero, the bending modulus would be zero by the classical theory. However, at the atomistic scale, the bond-angle effect on the interatomic interactions results in a finite bending modulus of graphene. For infinitesimal bending curvature $(\kappa \rightarrow 0)$, the bending modulus of planar graphene was predicted to be [26, 27]

$$
D_{0}=\frac{1}{2} V_{A}\left(r_{0}\right) \frac{\partial \bar{b}}{\partial \theta_{i j k}}=\frac{\sqrt{3}}{4} \frac{\partial V_{i j}}{\partial \cos \theta_{i j k}}
$$

where $V_{i j}$ is the interatomic potential as given in Eq. (22), $V_{A}\left(r_{0}\right)$ is the attractive part of the potential at the ground state, $\bar{b}$ is the bond order function given by Eq. (26), and $\theta_{i j k}$ is the angle between two atomic bonds $i-j$ and $i-k(k \neq i, j)$. For the $2^{\text {nd }}$ generation Brenner potential, the bending modulus was found to be $1.8 \mathrm{eV}-\AA^{2} /$ atom or equivalently, $0.11 \mathrm{nN}-\mathrm{nm}$ [26].

In the present study, we simulate bending of graphene by rolling planar graphene sheets into cylindrical tubes of different radii. Similar to the uniaxial stretch simulations, a rectangular computation cell with periodic boundary conditions is first selected and then rolled into a tube according to the deformation gradient in Eq. (21). As discussed in Section 2.5, for a particular

computational cell of size $L$, changing of the tube radius simultaneously changes the bending 
curvature and the stretch in the circumferential direction. To uncouple the bending and stretch, we set the tube radius $R=L / 2 \pi$ and use different computational cells with varying $L$ to get different radii and bending curvatures. In each simulation, the atoms are first displaced according to the deformation gradient in Eq. (21). Then, the total potential energy is minimized under the constraint that the tube radius does not change. To enforce this constraint, the atomic positions in one of the two sublattices of the graphene are fixed, while the atoms of the other sublattice are allowed to relax. In this way, the tube radius does not change during the energy minimization step. Consequently, all the 2D Green-Lagrange strain components are zero, and the normal curvature in the rolling direction is simply: $\kappa_{n}=\mathrm{K}_{11}=\frac{1}{R}$. The bending moment is then calculated by differentiating the strain energy density with respect to the curvature, and the tangent bending modulus is determined by the incremental relationship: $d M_{11}=D_{11} d \mathrm{~K}_{11}$. We note that several previous studies (e.g., Ref. 26) calculated strain energy of fully relaxed carbon nanotubes, which potentially included contributions from both bending and stretching. By applying the no-stretch constraint, the strain energy in the present study is purely bending relative to the planar graphene sheets at the ground state.

Figure 9 plots the strain energy per atom as a function of the bending curvature for graphene sheets rolled in the zigzag direction. Due to the constraint on the tube radius, the strain energy is higher than that of fully relaxed carbon nanotubes [26]. The obtained bending moment vs curvature is plotted in Figure 10. The bending moment increases almost linearly as the curvature increases in the range between $0.1 \mathrm{~nm}^{-1}$ to $2 \mathrm{~nm}^{-1}$, with slight nonlinearity at large curvatures. By a linear fitting with the first 10 data points, the bending modulus of graphene is obtained as $D_{11}=0.225 \mathrm{nN}-\mathrm{nm}$. The same bending modulus is obtained for rolling of graphene in the armchair direction. The bending modulus obtained from the present study is about twice of 
the bending modulus predicted by Eq. (30) in the previous studies [26, 27]. It is unclear what causes this discrepancy. The definition of the bending modulus in the present study (Eq. 15) is identical to Eq. (20) in Ref. [26] for infinitesimal bending curvature. While the bending modulus by Eq. (30) was found to be consistent with the strain energy of fully relaxed carbon nanotubes, it is not known how much stretch was induced by relaxation of the tube radius. A combination of bending and stretch could in principle lower the total strain energy of the nanotubes, which then leads to apparently lower bending moment and lower bending modulus. Furthermore, Eq. (30) appears to account for only one bond angle. Considering that each $\mathrm{C}-\mathrm{C}$ bond in the graphene lattice is associated with four bond angles (both $\theta_{i j k}$ and $\theta_{j i k}$ ), two for each carbon atom, we speculate that a summation over the two bond angles would result in doubling of the bending modulus $\left(2 D_{0}\right)$, which would then compare closely with the present MM simulations. Further studies are being conducted to better understand bending of graphene. Interestingly, the bending modulus obtained from the present MM simulations is surprisingly close to that predicted by an Ab initio study [34], as listed in Table I.

\section{Buckling of Graphene Ribbons}

A thin sheet tends to buckle under compression. In this section we simulate buckling of singleatomic-layer graphene ribbons under uniaxial compression. Similar to the MM simulations for uniaxial stretch, rectangular computational cells with periodic boundary conditions are used. Instead of the tensile strain $(\lambda=1+\varepsilon)$, uniaxial compressive strains $(\lambda=1-\varepsilon)$ are applied. An

initial perturbation with out-of-plane displacements of the atoms is introduced to trigger the buckling instability. It should be noted that the intrinsic rippling of the 2D graphene lattice [28] is not taken into account in the present simulations. 
Figure 11a shows the potential energy per atom as a function of the compressive strain in the zigzag direction for a graphene ribbon of width $L=1.97 \mathrm{~nm}$. Two curves are shown for comparison, one with initial perturbation and the other with in-plane deformation only. The two curves overlap each other at small strains but deviate from each other beyond a critical strain. The bifurcation due to buckling leads to a lower potential energy. Figure 11b plots the buckling amplitude (the maximum out-of-plane displacement) as a function of the compressive strain, from which the critical strain for the onset of buckling is clearly identified: $\varepsilon_{c}=0.0068$. The graphene ribbon remains planar before the critical strain, despite the initial perturbation. Beyond the critical strain, the buckling amplitude increases with the compressive strain. A snapshot of the deformed graphene ribbon at $\varepsilon=0.01$ (compressive) shows a nearly sinusoidal buckling profile (inset of Fig. 11b).

MM simulations are performed for graphene ribbons of various widths under uniaxial compression in different chiral directions. The critical strains for the onset of buckling are plotted in Fig. 12 for compression in the zigzag and armchair directions. It is found that the critical strain for buckling is insensitive to the chiral direction, but depends on the width of the graphene ribbon. The dependence is similar to that for the classical Euler buckling of thin plates [44], i.e., $\varepsilon_{c} \sim L^{-2}$. Noting that the critical strain for buckling is relatively small for the graphene ribbons considered in the present study, an isotropic, linear elastic behavior may be assumed for the buckling analysis. Similar to the classical Euler buckling [44], a linear analysis predicts that the critical load (force per unit length along the edge) for buckling of a graphene ribbon is

$$
P_{c}=\frac{4 \pi^{2} D}{L^{2}}
$$


where $D$ is the bending modulus and $L$ is the ribbon width. The critical nominal strain $\varepsilon_{c}$ for the onset of buckling is then

$$
\varepsilon_{c}=\frac{P_{c}}{C_{11}}=\frac{4 \pi^{2} D}{C_{11} L^{2}}
$$

where $C_{11}$ is taken to be the in-plane elastic modulus of graphene at infinitesimal strain. For comparison, we plot in Fig. 12 the prediction of the linear analysis using three sets of the elastic modulus and bending modulus of graphene (Table I). The elastic modulus and bending modulus obtained in Sections 4 and 5 of the present study give a prediction (linear analysis-1) that slightly overestimates the MM results. However, using the bending modulus by Eq. (30), the second line (linear analysis-2) significantly underestimates the critical strain. Interestingly, the linear prediction with the moduli from the $A b$ initio study [34] agrees closely with the MM results.

\section{Summary}

A theoretical framework of nonlinear continuum mechanics is developed for two-dimensional graphene sheets under both in-plane and bending deformation. Atomistic simulations by molecular mechanics are carried out for single-atomic-layer graphene sheets under uniaxial stretch, cylindrical bending, and buckling instability. It is found that graphene becomes highly nonlinear and anisotropic under finite-strain uniaxial stretch, and coupling between stretch and shear occurs except for stretching in the zigzag and armchair directions. The theoretical strength (nominal fracture strain and nominal fracture stress) of perfect graphene lattice also varies with the chiral direction of uniaxial stretch. By rolling graphene sheets into cylindrical tubes of various radii, the bending modulus of graphene is obtained, which differs from a previous 
prediction by a factor of 2. Buckling of graphene ribbons under uniaxial compression is simulated and the critical strain for the onset of buckling is compared to a linear buckling analysis. Future studies will investigate graphene sheets under combined bending and stretching as well as the effects of finite temperatures on the morphology and mechanical properties of graphene.

\section{Acknowledgments}

The authors gratefully acknowledge funding of this work by the US Department of Energy through Grant No. DE-FG02-05ER46230. 


\section{References}

1. T.A. Land, T. Michely, R.J. Behm, J.C. Hemminger, and G. Comsa, Stm Investigation of Single Layer Graphite Structures Produced on Pt(111) by Hydrocarbon Decomposition. Surface Science, 1992. 264(3): p. 261-270.

2. A. Nagashima, K. Nuka, H. Itoh, T. Ichinokawa, C. Oshima, and S. Otani, Electronic States of Monolayer Graphite Formed on Tic(111) Surface. Surface Science, 1993. 291(1-2): p. 93-98.

3. P.W. Sutter, J.I. Flege, and E.A. Sutter, Epitaxial graphene on ruthenium. Nature Materials, 2008. 7(5): p. 406-411.

4. A.L.V. de Parga, F. Calleja, B. Borca, M.C.G. Passeggi, J.J. Hinarejos, F. Guinea, and R. Miranda, Periodically rippled graphene: Growth and spatially resolved electronic structure. Physical Review Letters, 2008. 1(5).

5. C. Berger, Z.M. Song, T.B. Li, X.B. Li, A.Y. Ogbazghi, R. Feng, Z.T. Dai, A.N. Marchenkov, E.H. Conrad, P.N. First, and W.A. de Heer, Ultrathin epitaxial graphite: $2 D$ electron gas properties and a route toward graphene-based nanoelectronics. Journal of Physical Chemistry B, 2004. 108(52): p. 19912-19916.

6. C. Berger, Z.M. Song, X.B. Li, X.S. Wu, N. Brown, C. Naud, D. Mayou, T.B. Li, J. Hass, A.N. Marchenkov, E.H. Conrad, P.N. First, and W.A. de Heer, Electronic confinement and coherence in patterned epitaxial graphene. Science, 2006. 312(5777): p. 1191-1196.

7. I. Forbeaux, J.M. Themlin, and J.M. Debever, Heteroepitaxial graphite on 6H-SiC(0001): Interface formation through conduction-band electronic structure. Physical Review B, 1998. 58(24): p. 16396-16406. 
8. T. Ohta, A. Bostwick, T. Seyller, K. Horn, and E. Rotenberg, Controlling the electronic structure of bilayer graphene. Science, 2006. 313(5789): p. 951-954.

9. M.S. Dresselhaus and G. Dresselhaus, Intercalation compounds of graphite. Advances in Physics, 2002. 51(1): p. 1-186.

10. S. Horiuchi, T. Gotou, M. Fujiwara, T. Asaka, T. Yokosawa, and Y. Matsui, Single graphene sheet detected in a carbon nanofilm. Applied Physics Letters, 2004. 84(13): p. 2403-2405.

11. H. Shioyama, Cleavage of graphite to graphene. Journal of Materials Science Letters, 2001. 20(6): p. 499-500.

12. S. Stankovich, D.A. Dikin, G.H.B. Dommett, K.M. Kohlhaas, E.J. Zimney, E.A. Stach, R.D. Piner, S.T. Nguyen, and R.S. Ruoff, Graphene-based composite materials. Nature, 2006. 442(7100): p. 282-286.

13. L.M. Viculis, J.J. Mack, and R.B. Kaner, A chemical route to carbon nanoscrolls. Science, 2003. 299(5611): p. 1361-1361.

14. T.W. Ebbesen and H. Hiura, Graphene in 3-Dimensions - Towards Graphite Origami. Advanced Materials, 1995. 7(6): p. 582-586.

15. H. Hiura, T.W. Ebbesen, J. Fujita, K. Tanigaki, and T. Takada, Role of Sp(3) Defect Structures in Graphite and Carbon Nanotubes. Nature, 1994. 367(6459): p. 148-151.

16. X.K. Lu, M.F. Yu, H. Huang, and R.S. Ruoff, Tailoring graphite with the goal of achieving single sheets. Nanotechnology, 1999. 10(3): p. 269-272.

17. K.S. Novoselov, A.K. Geim, S.V. Morozov, D. Jiang, Y. Zhang, S.V. Dubonos, I.V. Grigorieva, and A.A. Firsov, Electric field effect in atomically thin carbon films. Science, 2004. 306(5696): p. 666-669. 
18. H.V. Roy, C. Kallinger, B. Marsen, and K. Sattler, Manipulation of graphitic sheets using a tunneling microscope. Journal of Applied Physics, 1998. 83(9): p. 4695-4699.

19. Y.B. Zhang, J.P. Small, W.V. Pontius, and P. Kim, Fabrication and electric-fielddependent transport measurements of mesoscopic graphite devices. Applied Physics Letters, 2005. 86(7).

20. K.S. Novoselov, D. Jiang, F. Schedin, T.J. Booth, V.V. Khotkevich, S.V. Morozov, and A.K. Geim, Two-dimensional atomic crystals. Proceedings of the National Academy of Sciences of the United States of America, 2005. 102(30): p. 10451-10453.

21. A.K. Geim and K.S. Novoselov, The rise of graphene. Nature Materials, 2007. 6(3): p. 183-191.

22. L.D. Landau, E.M. Lifshits, L.P. Pitaevskii, J.B. Sykes, and M.J. Kearsley, Statistics physics, Part I. 1980, Oxford: Pergamon.

23. N.D. Mermin, Crystalline Order in Two Dimensions. Physical Review, 1968. 176(1): p. 250.

24. J.C. Meyer, A.K. Geim, M.I. Katsnelson, K.S. Novoselov, T.J. Booth, and S. Roth, The structure of suspended graphene sheets. Nature, 2007. 446(7131): p. 60-63.

25. D.R. Nelson, T. Piran, and S. Weinberg, Statistical mechanics of membranes and surfaces. 2004, Singapore: World Scientific Pub.

26. M. Arroyo and T. Belytschko, Finite crystal elasticity of carbon nanotubes based on the exponential Cauchy-Born rule. Physical Review B, 2004. 69(11).

27. Y. Huang, J. Wu, and K.C. Hwang, Thickness of graphene and single-wall carbon nanotubes. Physical Review B, 2006. 74(24). 
28. A. Fasolino, J.H. Los, and M.I. Katsnelson, Intrinsic ripples in graphene. Nature Materials, 2007. 6(11): p. 858-861.

29. J.S. Bunch, A.M. van der Zande, S.S. Verbridge, I.W. Frank, D.M. Tanenbaum, J.M. Parpia, H.G. Craighead, and P.L. McEuen, Electromechanical resonators from graphene sheets. Science, 2007. 315(5811): p. 490-493.

30. D. Garcia-Sanchez, A.M. van der Zande, A.S. Paulo, B. Lassagne, P.L. McEuen, and A. Bachtold, Imaging mechanical vibrations in suspended graphene sheets. Nano Letters, 2008. 8(5): p. 1399-1403.

31. I.W. Frank, D.M. Tanenbaum, A.M. Van der Zande, and P.L. McEuen, Mechanical properties of suspended graphene sheets. Journal of Vacuum Science \& Technology B, 2007. 25(6): p. 2558-2561.

32. M. Poot and H.S.J. van der Zant, Nanomechanical properties of few-layer graphene membranes. Applied Physics Letters, 2008. 92(6).

33. C. Lee, X.D. Wei, J.W. Kysar, and J. Hone, Measurement of the elastic properties and intrinsic strength of monolayer graphene. Science, 2008. 321(5887): p. 385-388.

34. K.N. Kudin, G.E. Scuseria, and B.I. Yakobson, $C 2 F, B N$, and $C$ nanoshell elasticity from ab initio computations. Physical Review B, 2001. 64(23).

35. D. Sanchez-Portal, E. Artacho, J.M. Soler, A. Rubio, and P. Ordejon, Ab initio structural, elastic, and vibrational properties of carbon nanotubes. Physical Review B, 1999. 59(19): p. $12678-12688$.

36. G. Van Lier, C.V. Alsenoy, V.V. Doren, and P. Geerlings, Ab initio study of the elastic properties of single-walled carbon nanotubes and graphene. Chemical Physics Letters, 2000. 326(1-2): p. 181-185. 
37. Q. Wang, Effective in-plane stiffness and bending rigidity of armchair and zigzag carbon nanotubes. International Journal of Solids and Structures, 2004. 41(20): p. 5451-5461.

38. D. Caillerie, A. Mourad, and A. Raoult, Discrete homogenization in graphene sheet modeling. Journal of Elasticity, 2006. 84(1): p. 33-68.

39. R. Khare, S.L. Mielke, J.T. Paci, S.L. Zhang, R. Ballarini, G.C. Schatz, and T. Belytschko, Coupled quantum mechanical/molecular mechanical modeling of the fracture of defective carbon nanotubes and graphene sheets. Physical Review B, 2007. 75(7).

40. F. Liu, P.M. Ming, and J. Li, Ab initio calculation of ideal strength and phonon instability of graphene under tension. Physical Review B, 2007. 76(6).

41. C.D. Reddy, S. Rajendran, and K.M. Liew, Equilibrium configuration and continuum elastic properties of finite sized graphene. Nanotechnology, 2006. 17(3): p. 864-870.

42. X. Zhang, K. Jiao, P. Sharma, and B.I. Yakobson, An atomistic and non-classical continuum field theoretic perspective of elastic interactions between defects (force dipoles) of various symmetries and application to graphene. Journal of the Mechanics and Physics of Solids, 2006. 54(11): p. 2304-2329.

43. J. Zhou and R. Huang, Internal lattice relaxation of single-layer graphene under in-plane deformation. Journal of the Mechanics and Physics of Solids, 2008. 56: p. 1069.

44. S. Timoshenko and S. Woinowsky-Krieger, Theory of Plates and Shells. 2nd ed. 1987, New York: McGraw-Hill.

45. M. Arroyo and T. Belytschko, Finite element methods for the non-linear mechanics of crystalline sheets and nanotubes. International Journal for Numerical Methods in Engineering, 2004. 59(3): p. 419-456. 
46. M.P. do Carmo, Differential Geometry of Curves snd Surfaces. 1976, Englewood Cliffs, NJ: Prentice-Hall.

47. J.E. Marsden and T.J.R. Hughes, Mathematical Foundation of Elasticity. 1983, Englewood Cliffs, NJ: Prentice-Hall.

48. D.W. Brenner, Empirical potential for hydrocarbons for use in simulating the chemical vapor deposition of diamond films. Phys. Rev. B, 1990. 42: p. 9458-9471.

49. D.W. Brenner, O.A. Shenderova, J.A. Harrison, S.J. Stuart, B. Ni, and S.B. Sinnott, $A$ second-generation reactive empirical bond order (REBO) potential energy expression for hydrocarbons. Journal of Physics-Condensed Matter, 2002. 14(4): p. 783-802.

50. S.S. Terdalkar, S.L. Zhang, J.J. Rencis, and K.J. Hsia, Molecular dynamics simulations of ion-irradiation induced deflection of 2D graphene films. International Journal of Solids and Structures, 2008. 45(13): p. 3908-3917.

51. R. Clausius, On a mechanical theory applicable to heat. Philosophical Magazine, 1870. 40: p. $122-127$.

52. J. Nocedal. Updating Quasi-Newton Matrices with Limited Storage. Mathematics of Computation, 1980. 35: p. 773-782.

53. Y.N. Gartstein, A.A. Zakhidov, R.H. Baughman, Mechanical and electromechanical coupling in carbon nanotube distortions. Phys. Rev. B, 2003. 68: 115415.

54. H.Y. Liang and M. Upmanyu, Axial-strain-induced torsion in single-walled carbon nanotubes. Physical Review Letters, 2006. 96: 165501. 
Table I. Comparison of elastic moduli of single-atomic-layer graphene under infinitesimal deformation (Young's modulus $Y=C_{11}-C_{21}^{2} / C_{11}$ and Poisson's ratio $v=C_{21} / C_{11}$ )

\begin{tabular}{c|ccccc}
\hline & $C_{11}(\mathrm{~N} / \mathrm{m})$ & $C_{21}(\mathrm{~N} / \mathrm{m})$ & $Y(\mathrm{~N} / \mathrm{m})$ & $v$ & $\begin{array}{c}D \\
(\mathrm{nN}-\mathrm{nm})\end{array}$ \\
\hline Present study (MM) & 288.8 & 114.9 & 243 & 0.398 & 0.225 \\
Arroyo and Belytschko [26] & 288 & 114.5 & 243 & 0.397 & 0.11 \\
Ab initio [34] & 353 & 52.6 & 345 & 0.149 & 0.238 \\
\hline
\end{tabular}




\section{Figures}

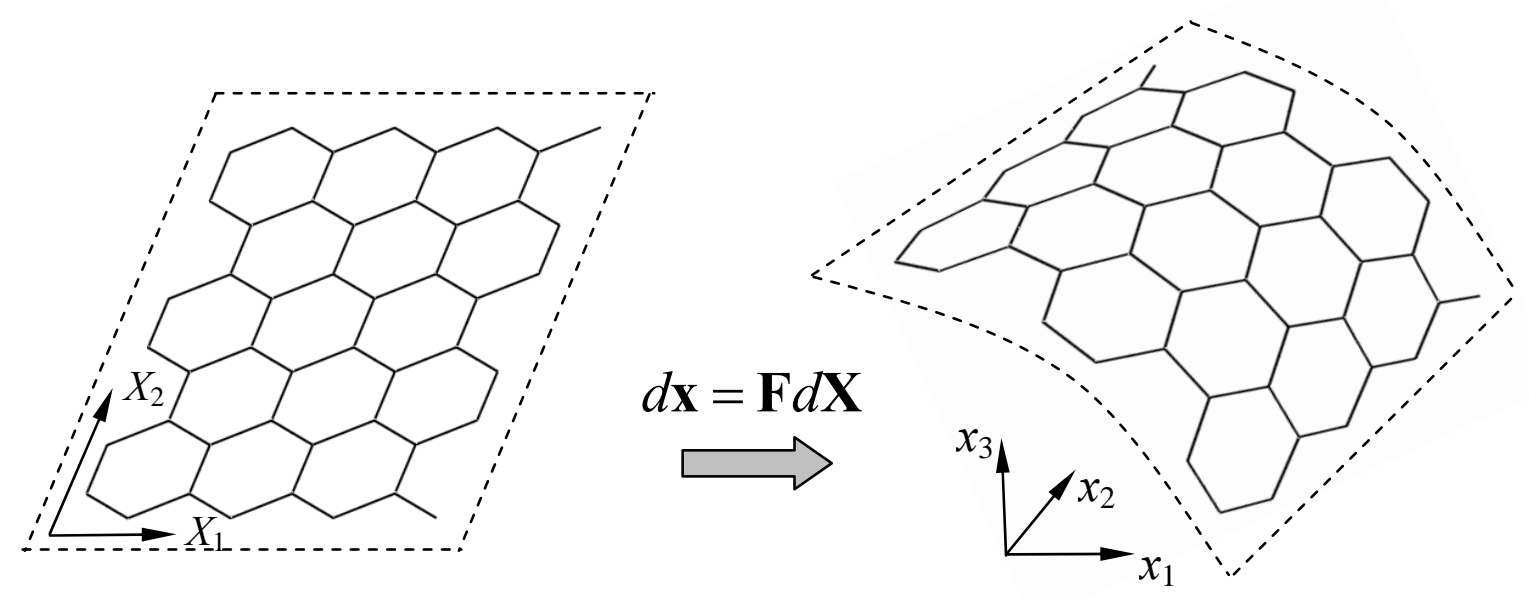

Figure 1. Schematic illustration of a 2D graphene sheet before and after deformation. 
Qiang Lu and Rui Huang, Nonlinear mechanics of single-atomic-layer graphene sheets

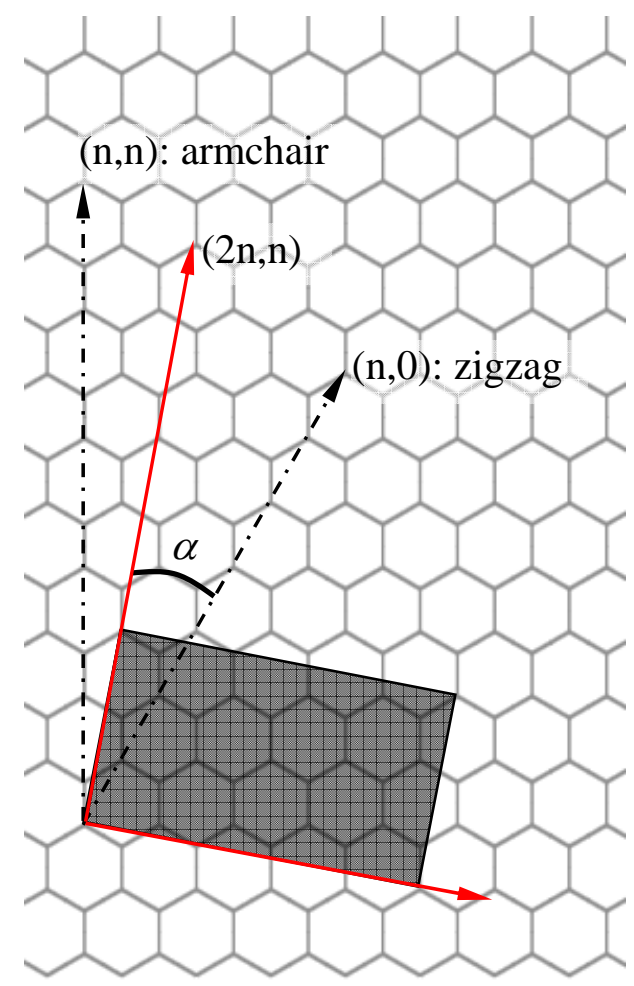

Figure 2. Illustration of a rectangular unit cell of graphene lattice with a particular chiral direction. 
Qiang Lu and Rui Huang, Nonlinear mechanics of single-atomic-layer graphene sheets
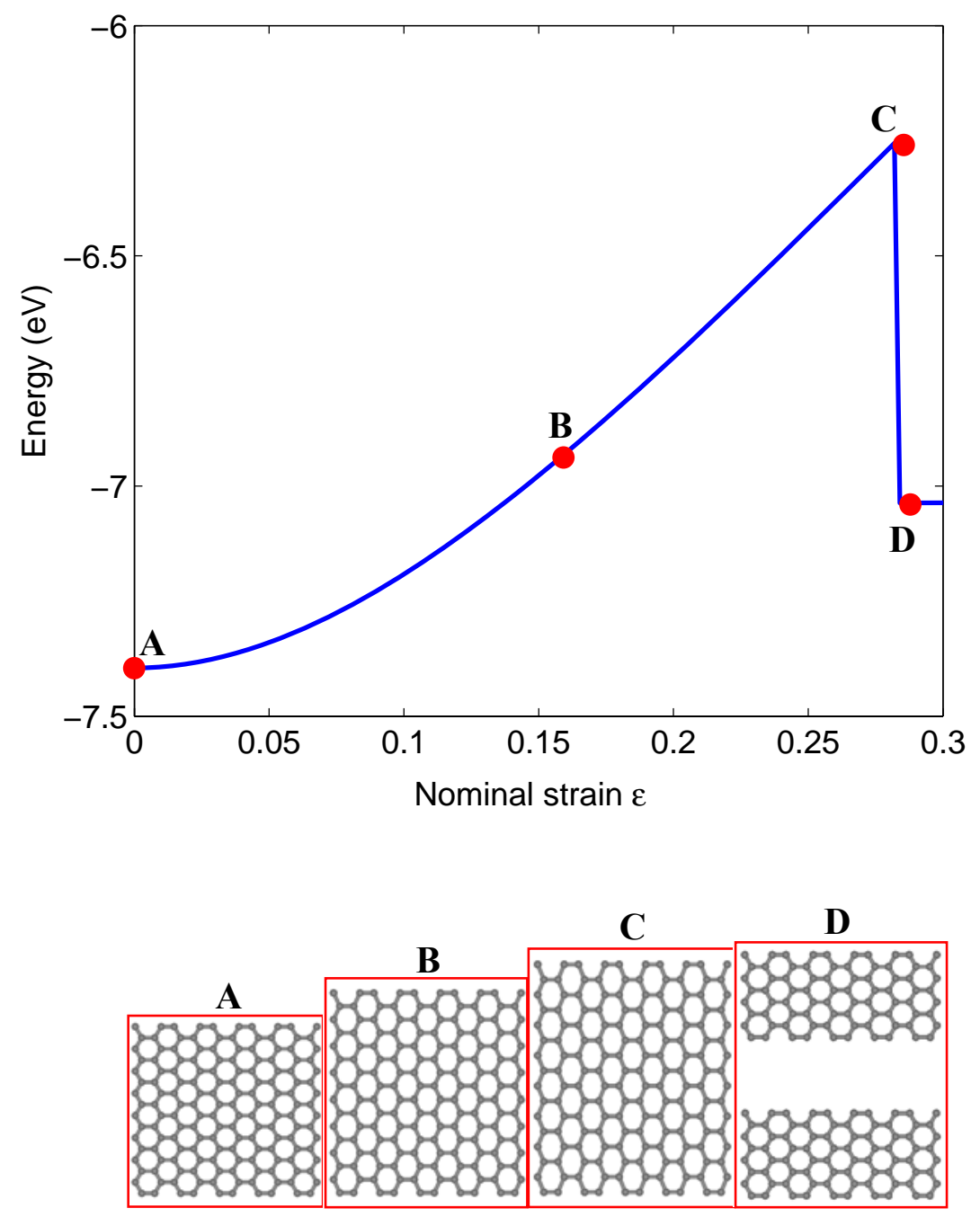

Figure 3. Energy per atom vs nominal strain of a single-atomic-layer graphene sheet under uniaxial stretch in the zigzag direction $(\alpha=0)$, with snapshots of the equilibrium atomic structures at $\varepsilon=0(\mathrm{~A}), 0.16(\mathrm{~B}), 0.282(\mathrm{C})$, and $0.284(\mathrm{D})$. 
Qiang Lu and Rui Huang, Nonlinear mechanics of single-atomic-layer graphene sheets
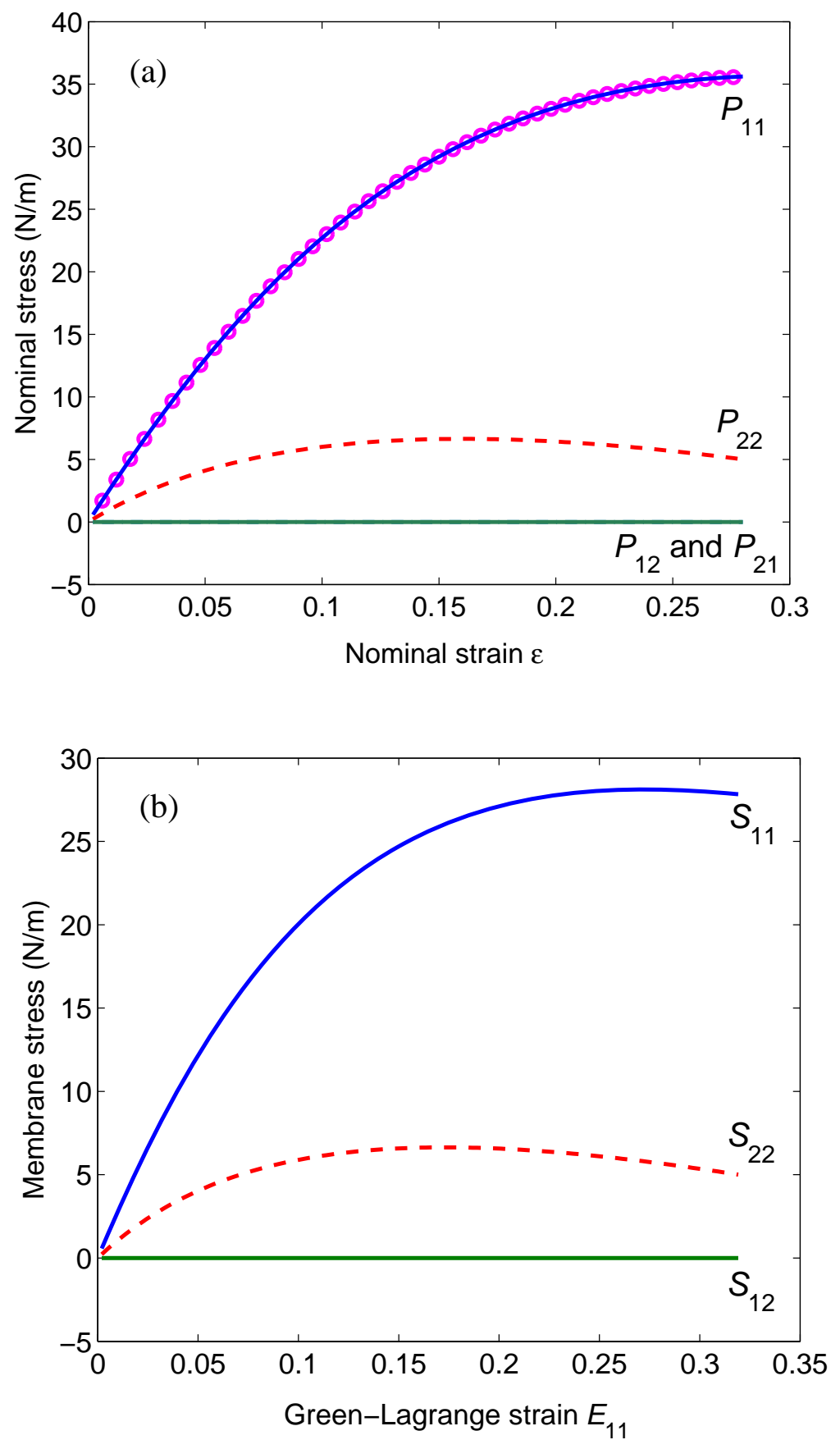

Figure 4. (a) Nominal stresses vs nominal strain and (b) Second Piola-Kirchhoff membrane stresses vs Green-Lagrange strain for a single-atomic-layer graphene sheet under uniaxial stretch in the zigzag direction. The nominal stress $P_{11}$ is calculated by both the energy method (open circles) and the viral stress method (solid line). 
Qiang Lu and Rui Huang, Nonlinear mechanics of single-atomic-layer graphene sheets

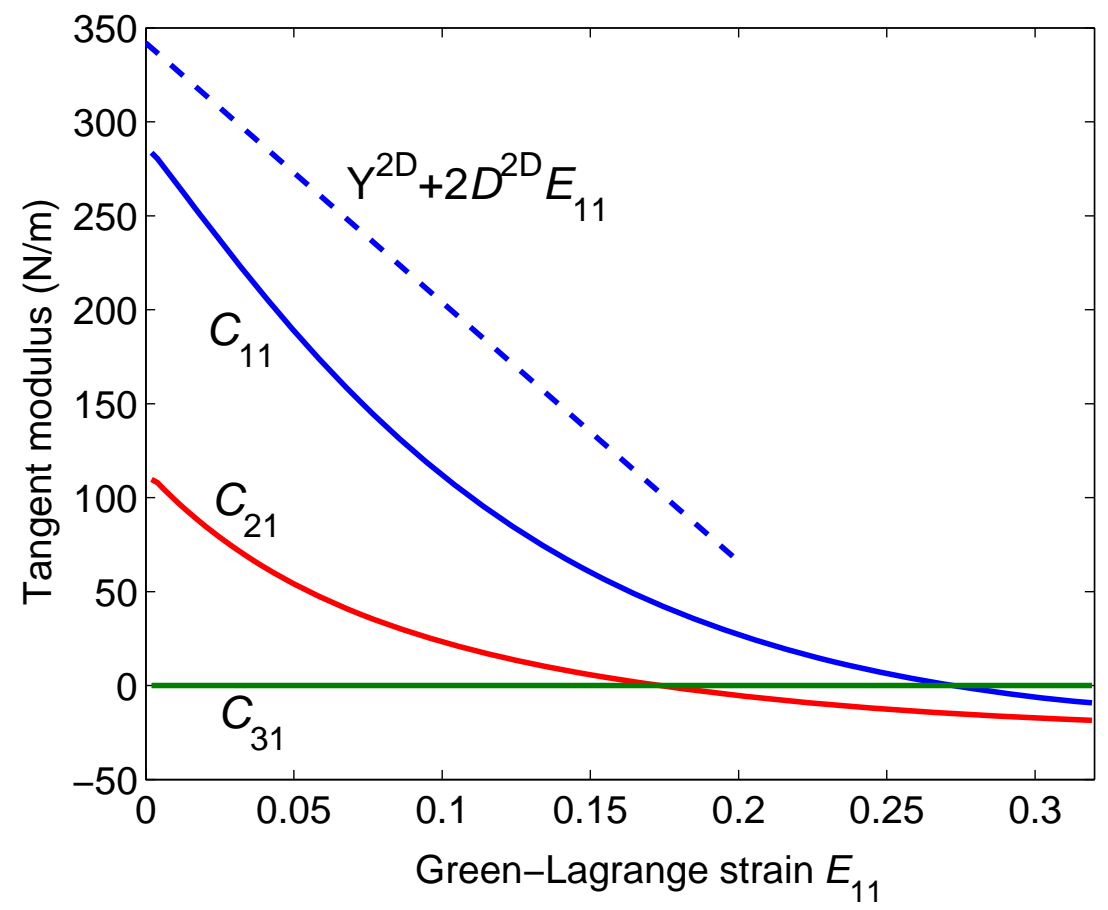

Figure 5. Tangent elastic moduli of a single-atomic-layer graphene sheet under uniaxial stretch in the zigzag direction. The dashed line is the tangent Young's modulus from Eq. (29) as suggested in Ref. [33]. 
Qiang Lu and Rui Huang, Nonlinear mechanics of single-atomic-layer graphene sheets

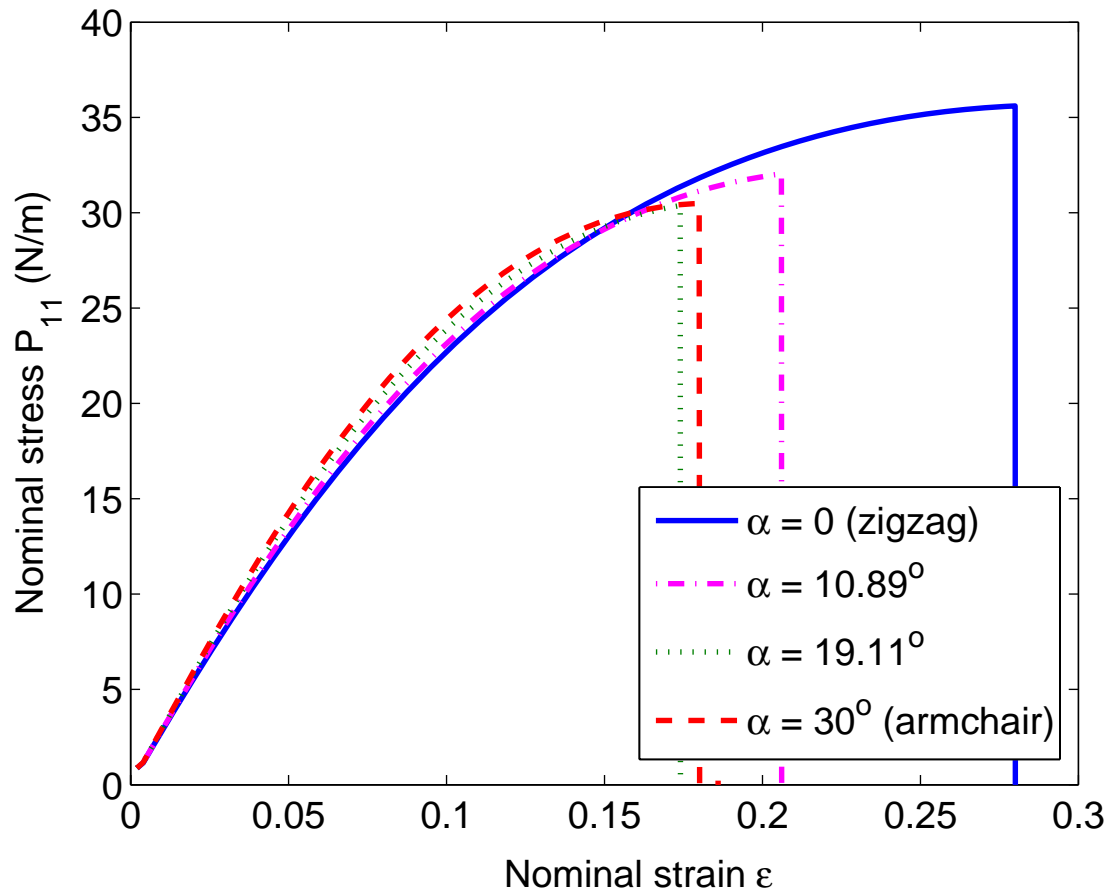

Figure 6. Nominal stress vs nominal strain for single-atomic-layer graphene sheets under uniaxial stretch along different chiral directions. 
Qiang Lu and Rui Huang, Nonlinear mechanics of single-atomic-layer graphene sheets
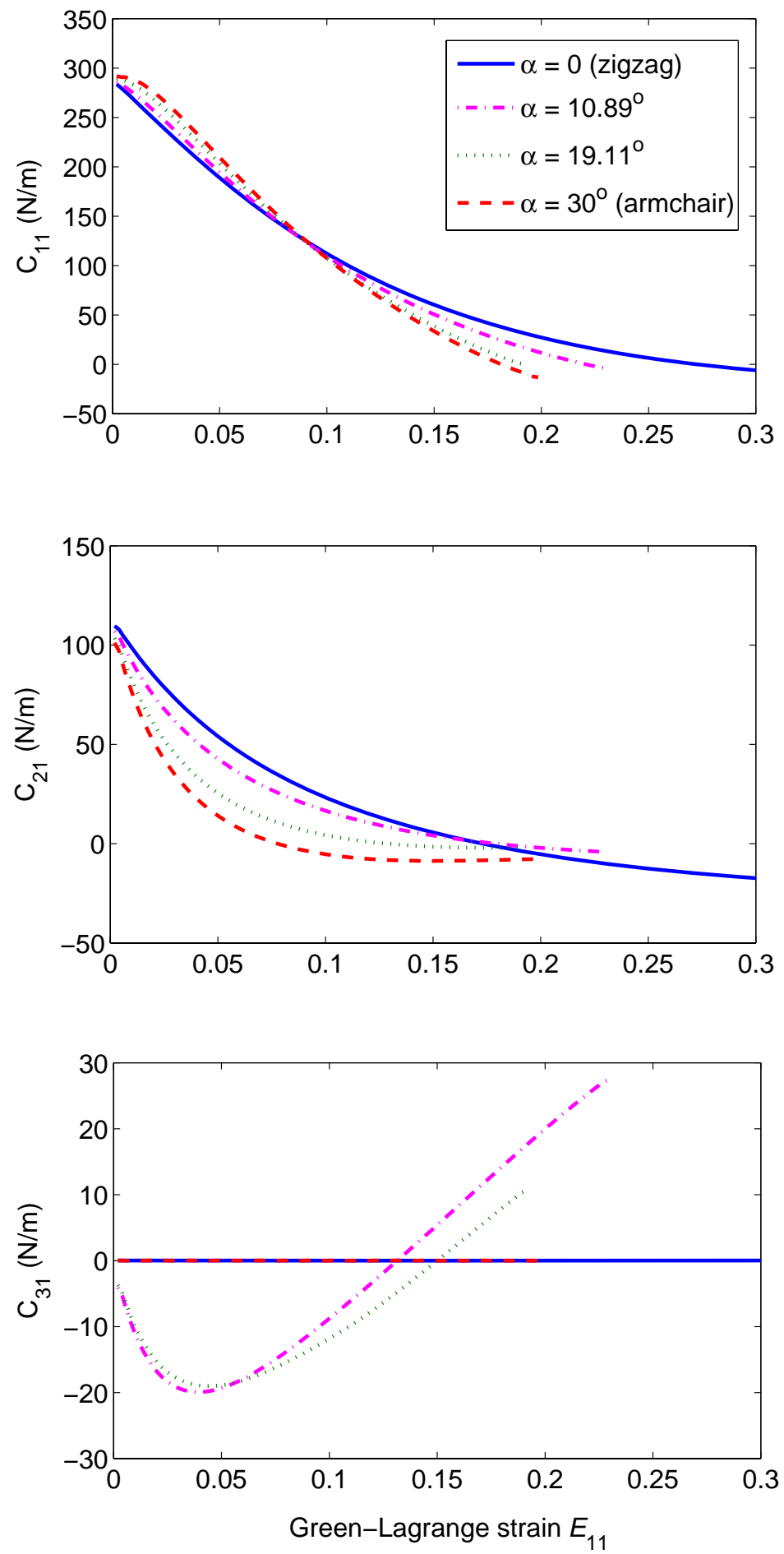

Figure 7. Tangent elastic moduli $C_{11}, C_{21}$ and $C_{31}$ of single-atomic-layer graphene sheets under uniaxial stretch along different chiral directions. 
Qiang Lu and Rui Huang, Nonlinear mechanics of single-atomic-layer graphene sheets

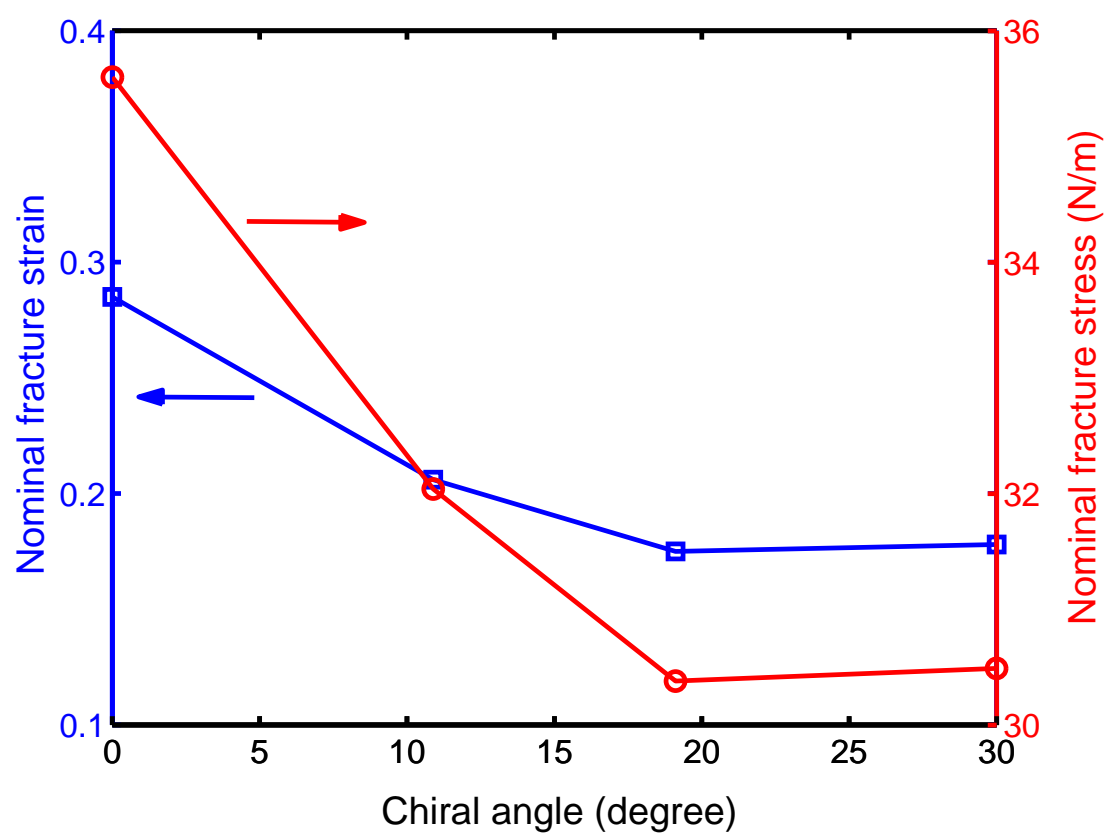

Figure 8. Nominal fracture strain and stress of single-atomic-layer graphene sheets under uniaxial stretch along different chiral directions. 
Qiang Lu and Rui Huang, Nonlinear mechanics of single-atomic-layer graphene sheets

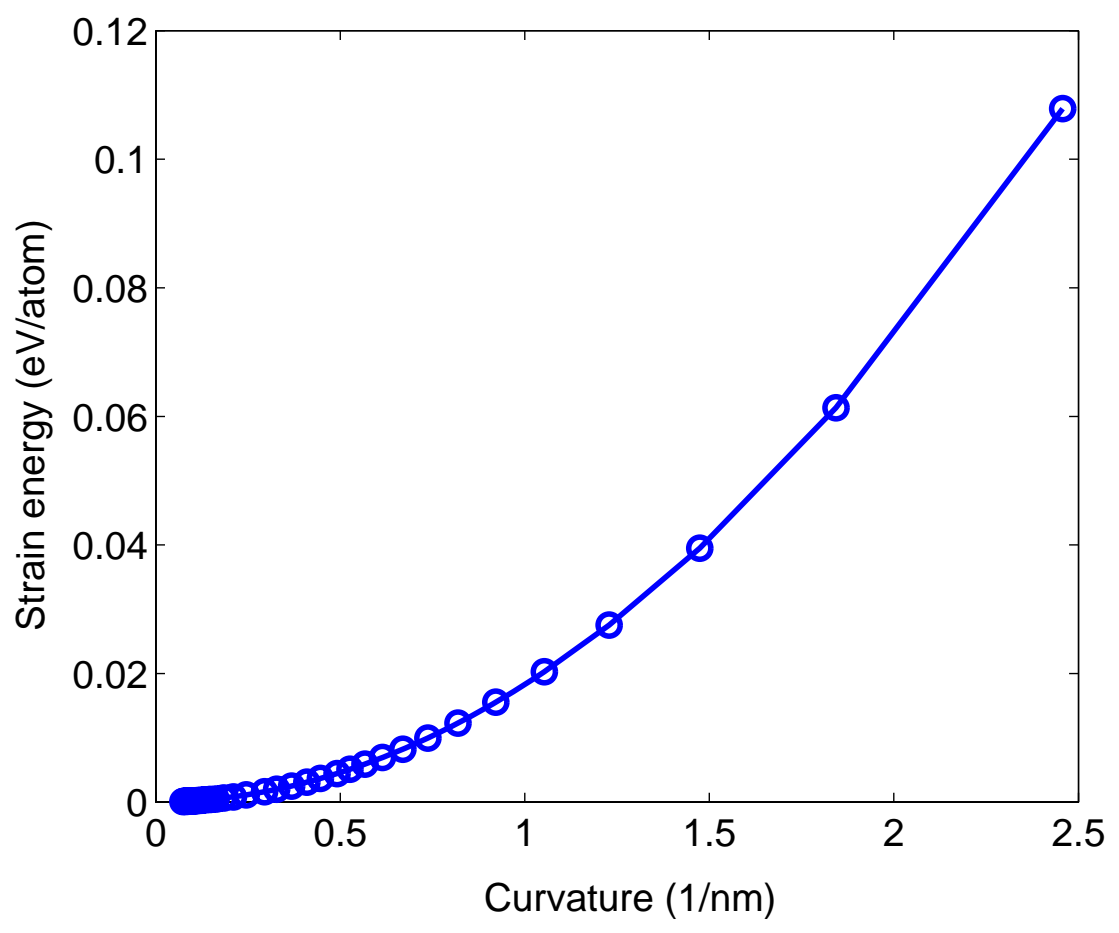

Figure 9. Strain energy per atom of single-atomic-layer graphene sheets rolled into cylindrical tubes of different curvatures $(\kappa=1 / R)$ along the zigzag direction; the stretch in the circumferential and axial directions of the tubes is constrained so that the 2D Green-Lagrange strain components are all zero. 
Qiang Lu and Rui Huang, Nonlinear mechanics of single-atomic-layer graphene sheets

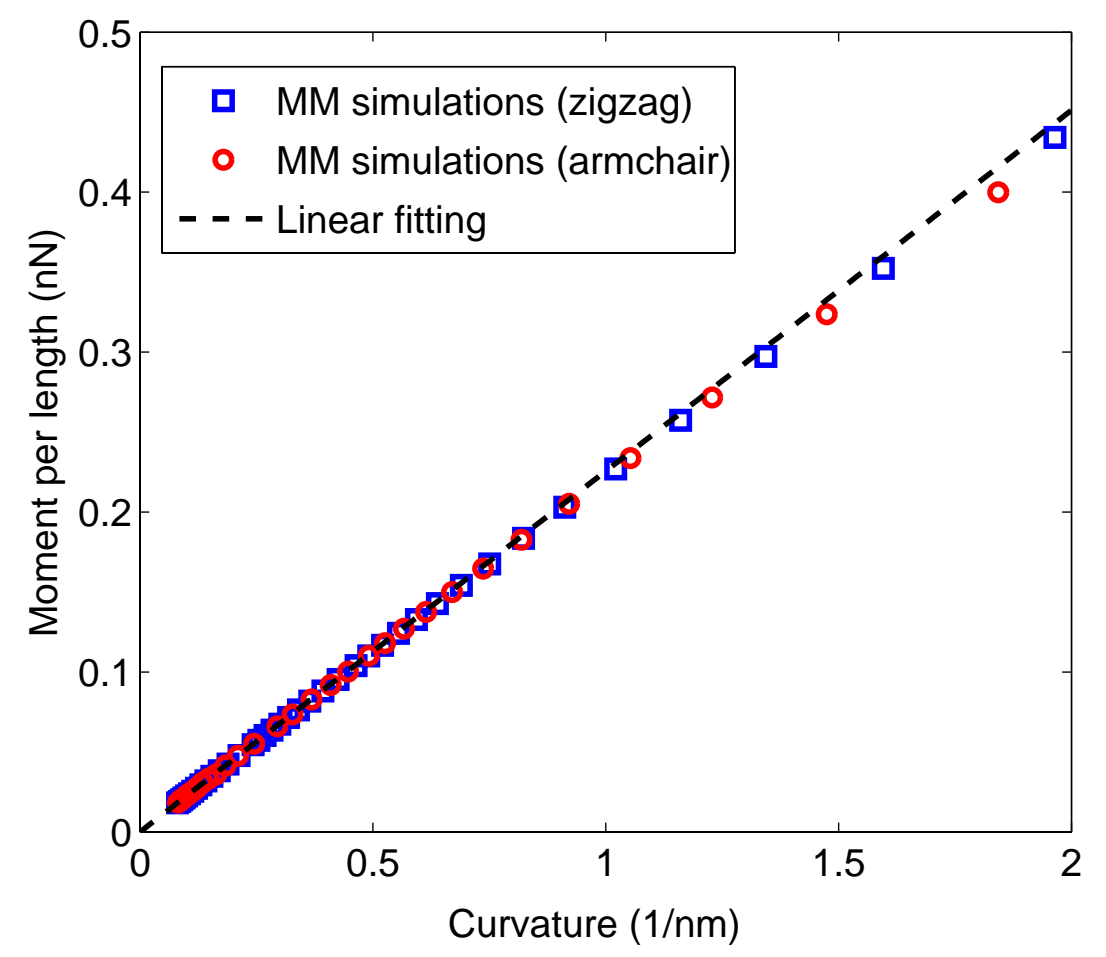

Figure 10. Bending moment vs curvature for cylindrical bending of single-atomic-layer graphene sheets in the zigzag and armchair directions. 
Qiang Lu and Rui Huang, Nonlinear mechanics of single-atomic-layer graphene sheets
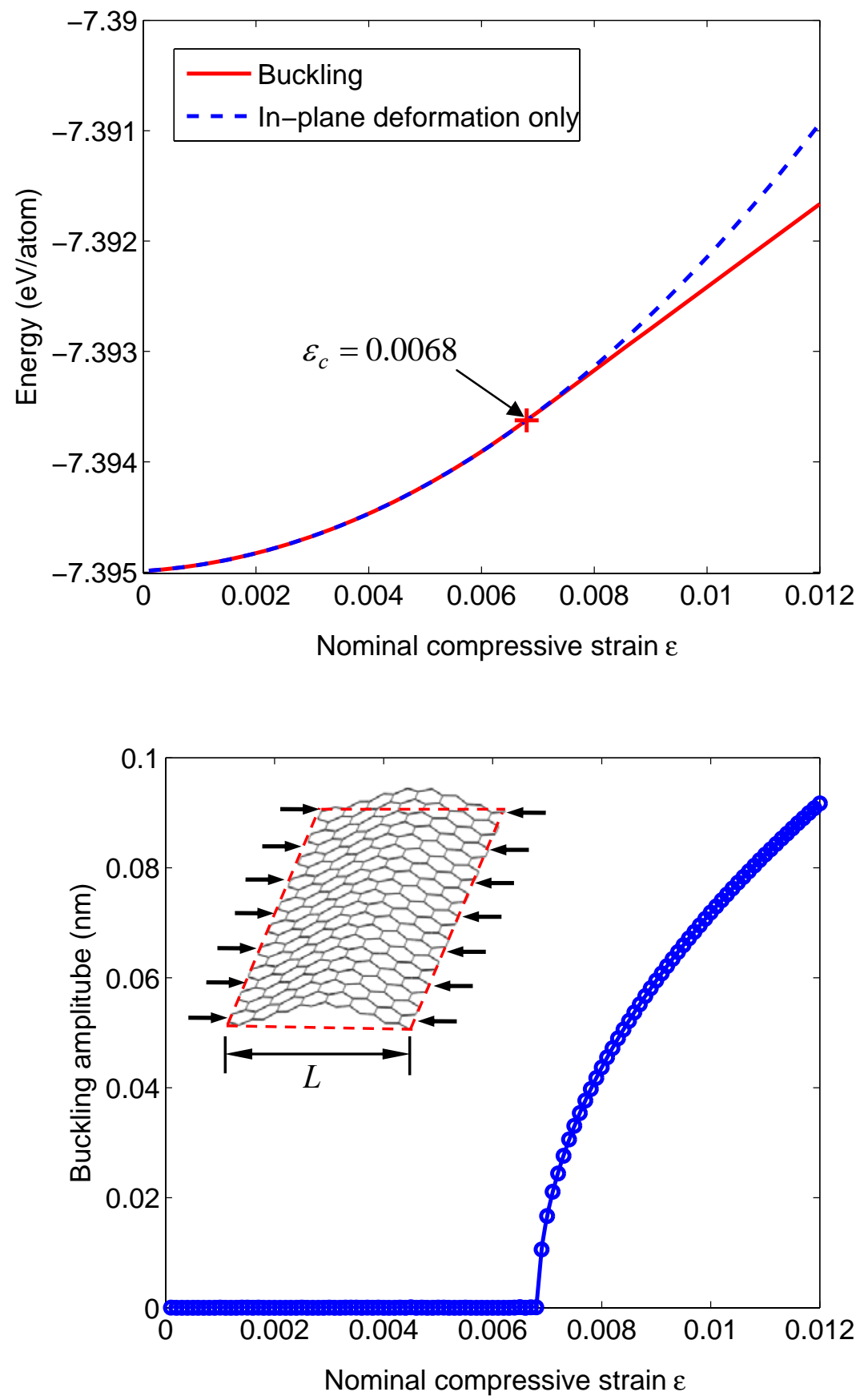

Figure 11. (a) Energy per atom and (b) buckling amplitude vs nominal compressive strain for a single-atomic-layer graphene ribbon $(L=1.97 \mathrm{~nm})$. For comparison, the dashed line in (a) shows the energy for in-plane deformation only. The inset in (b) shows a buckled graphene ribbon at $\varepsilon=0.01$. 
Qiang Lu and Rui Huang, Nonlinear mechanics of single-atomic-layer graphene sheets

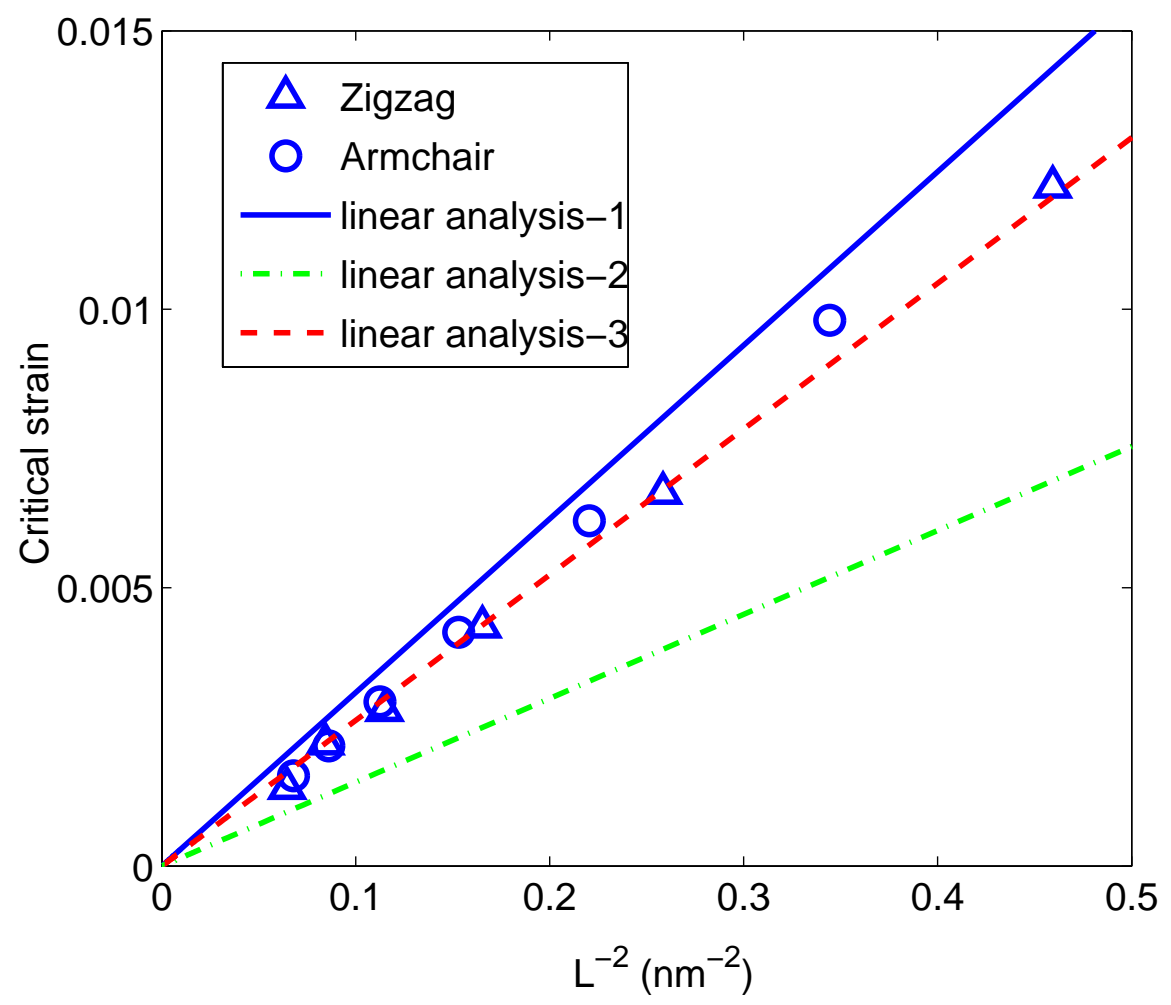

Figure 12. Critical strain for onset of buckling for single-atomic-layer graphene ribbons under uniaxial compression in the zigzag and armchair directions by molecular mechanics simulations, in comparison with the predictions by a linear buckling analysis using the elastic moduli listed in Table I. 